\title{
A comparison among modal parameter extraction methods
}

\author{
Abdel Kader Zrayka ${ }^{1} \cdot$ Emiliano Mucchi $^{1}$
}

(c) Springer Nature Switzerland AG 2019

\begin{abstract}
An effective comparison among several methods for extraction of the modal parameters from the frequency response functions measurements is presented in this research. In particular, several curve fitting methods, which are the peak amplitude method, the circle fit method, the least square complex exponential method, the eigensystem realization algorithm method and the rational fraction polynomial method were implemented in Matlab environment and compared in terms of natural frequencies, modal damping and mode shapes. Measurements were performed on a carcass of the gearbox in free-free condition. A hammer has been used with a periodic impulsive excitation signal. The natural frequencies values obtained by all methods were very similar and the differences between the results were insignificant. The peak amplitude and the circle fit gave good results for the damping ratios. The rational fraction polynomial method did the best job in detecting the damping and frequency values. The results obtained by the least square complex exponential method and the eigensystem realization algorithm method were reasonable for both frequency and damping.
\end{abstract}

Keywords Eigensystem realization algorithm · Least square complex exponential · Modal analysis methods · Rational fraction polynomial $\cdot$ Implementation modal analysis by Matlab ${ }^{\circledR}$

\begin{tabular}{|c|c|c|c|}
\hline \multicolumn{2}{|c|}{ List of symbols } & $q$ & Number of force input locations \\
\hline \multirow{2}{*}{$a_{k}$} & \multirow{2}{*}{$\begin{array}{l}\text { Coefficients of the numerator polynomial of } \\
\alpha(j \omega)\end{array}$} & $t$ & Time \\
\hline & & $\Delta t$ & Simple time \\
\hline $\boldsymbol{A}$ & $\begin{array}{l}\text { State transition matrix characterizing the } \\
\text { dynamics of the system }\end{array}$ & {$[\boldsymbol{U}]$} & $\begin{array}{l}\text { Orthogonal (or unitary) matrix of left singular } \\
\text { vectors, in the SVD technique }\end{array}$ \\
\hline \multirow{3}{*}{$\begin{array}{l}A_{p q}^{r} \\
b_{k}\end{array}$} & Modal constant for mode $r$ & {$[\boldsymbol{V}]$} & Orthogonal (or unitary) matrix of right singular \\
\hline & Coefficients of the denominator polynomial of & & vectors, in the SVD technique \\
\hline & $\alpha(j \omega)$ & $x(t)$ & State vector \\
\hline B & Input matrix & $y(t)$ & Output vector \\
\hline C & Output matrix & $\alpha_{p q}(j \omega)$ & Receptance FRF (mm/N) \\
\hline D & The direct input-output transmission matrix & $\psi_{u}$ & Vector space unscaled eigenvector \\
\hline$f_{r}$ & Natural frequency of mode $r(\mathrm{~Hz})$ & $\psi$ & Mode shape in term of physical coordinate of \\
\hline$[H]$ & Hankel matrix & & the system \\
\hline$h_{p q}(t)$ & Impulse response function corresponding to & $\zeta_{r}$ & Damping factor of mode $r$ \\
\hline & $\alpha_{p q}(j \omega)$ & $\theta$ & Phase angle (rad) \\
\hline$\widetilde{h_{p q}}$ & Vector formed by $h_{p q}(t)$ elements & $\Delta \theta$ & Variation of phase angle between $\theta_{i}$ and $\theta_{i+1}$ \\
\hline$i^{p 4}$ & Index & $\lambda_{r}$ & System pole of mode $r$ \\
\hline$j \omega$ & Laplace variable & $\omega$ & Circular frequency (rad/s) \\
\hline$N$ & Number of degrees-of-freedom of the system & $\omega_{r}$ & Natural frequency of mode $\mathrm{r}(\mathrm{rad} / \mathrm{s})$ \\
\hline$p$ & Number of measured response locations & {$[\Sigma]$} & Singular value matrix \\
\hline
\end{tabular}

Abdel Kader Zrayka, zryblk@unife.it; Emiliano Mucchi, mccmln@unife.it | Department of Engineering, University of Ferrara, Via Saragat 1, 44122 Ferrara, Italy. 


\section{Operators}

$[\ldots]^{T} \quad$ Transpose matrix

$[\ldots]^{-1} \quad$ Inverse matrix

$\mathcal{F}^{-1} \quad$ Inverse Fourier trasformation

\section{Abbreviations}

FRF Frequency response function

CFM Circle fit method

ERAM Eigensystem realization algorithm method

IRF Impulse response function

MDOF Multi degree of freedom

LSCEM Least square complex exponential method

PPM Peak amplitude or pick picking method

RFPM Rational fraction polynomial method

SDOF Single degree of freedom

\section{Introduction}

Nowadays, technologies play a great role in human life, advancing the ways of living and working but engineers still have many issues to solve in various filed of technology. The vibration of mechanical structure remains one of the most studied problems due to the difficulty to completely avoid vibration in many applications.

Currently, a large number of algorithms and literature for curve fitting structural data is available; consequently, the determination of the optimal method has become difficult for each situation. Hence, the main objective of this paper is the comparison among several modal parameter extraction algorithms from response vibration measurements in order to highlight not only their pros and cons. Moreover, this article focuses on how these methods can be implemented in a simple way in order to find the natural frequencies and the damping ratios to make them easy to use in the dynamic environment. As these authors are aware, in the literature the detailed explanation on the steps used to implement these methods or the tricks necessary to achieve good results are not provided. This mostly happens when the least square complex exponential method, the eigensystem realization algorithm method and the rational fraction polynomial method are used. on the other hand, the peak amplitude and the circle fit methods are well clarified and achieved. Therefore, it was decided to use the latters to get initial estimation of the modal parameters. Furthermore, the implementation of these methods highly differs between authors. In this work, a simplified and efficient implementation on Matlab ๑ is illustrated, in order to extract modal parameters. The basic problem in experimental modal analysis is to extract the natural frequency and especially the damping ratio of dynamical structure. With this knowledge, a theoretical finite element models [1] can validate and gain a better comprehension of corresponding structure. A further widely used application of these modal parameters is to control the health of a structure. The modal analysis methods can be separated in two leading categories which are the time domain and the frequency domain methods [2]; the earliest method works in the frequency domain and is classified as an SDOF algorithm such as the peak amplitude method (PPM) [3] and the circle fit method (CFM) [4]. These methods give errors in results, particularly in the damping estimation, where the modes are close to each other and coupled. In addition, the SDOF algorithm should not be used when the data contains noise around the resonance [5]. When the MDOF algorithms operate in the time domain and frequency domain methods, data are managed by assorting the FRFs in the domain. The least square complex exponential method (LSCEM) is considered one of the fast time domain methods. [6, 7]. This method uses the FRFs as an input in spite the fact that it works in the time domain. The LSCEM uses the Least Square Method to find the modal parameters. The eigensystem realization algorithm method (ERAM) is another major MDOF time domain approach $[8,9]$. This method uses the Singular Values Decomposition of the so-called Hankel Matrix which is usually a matrix with a high number of rows and columns; for such a reason this algorithm is computationally complex [10]. One more MDOF is the rational fraction polynomial method (RFPM) [11]; it works in the frequency domain and it uses the Least Square technique to minimize the error function. For more information concerning modal analysis techniques can be addressed in Ref $[12,13]$.

In the frequency domain method, the lower number of order is, the more accurate the results are. However, they have specific problems related to the fast Fourier transform (FFT) analysis [14, 15], such as the leakage [16]. Furthermore, the frequency domain methods determine the frequency response function, but this task usually requires the input excitation data to be detailed. In time domain, signals are monitored by the time domain methods using output responses only. Time domain methods are considered very useful for experimental tests due to this characteristic. Another useful property of time domain method is the ability to recognize two modes when they are very close one to each other. In general, the time domain work better than the frequency domain when the damping is low, and the frequency domain estimators work better for high damping, as demonstrated in $[17,18]$.

It has always been a difficult task to define the poles of a system, including the damping and the frequency, from the response vibration responses. In this work, the carcass, one of the gear box components, was separately considered for a better estimation of the FRFs [19]. The measurements were carried out after adding 


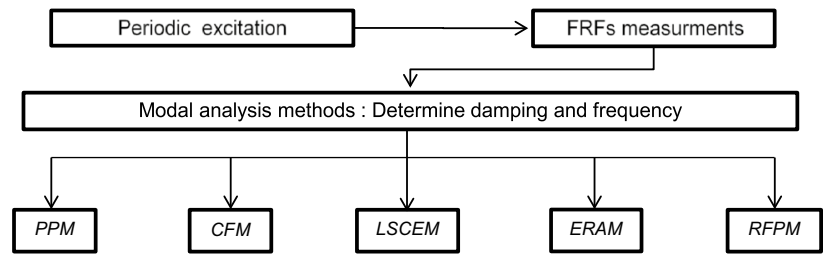

Fig. 1 Analysis procedure's flowchart

a constrained layer to the carcass of gear box to make the damping degree similar to the free-free condition. The structure was excited by a hammer with a periodic impulse. The residues or the zeros of the system Frequency Response Function (FRFs) can be equivalently determined using the periodic response. The original contribution of this study is related to the implementation of several methods in Maltab ${ }^{\circledR}$ to extract their modal parameters in order to then compare their results, discuss the limits of each method and its suitable domain of employment. For the comparison, the following common methods of modal analysis were used: peak amplitude methods (PPM), circle fit method (CFM), least square complex exponential method (LSCEM), eigen system realization algorithm method (ERAM) and rational fraction polynomial method (RFPM). The process followed to determine the poles is shown in the flowchart of Fig. 1.

\section{Background and algorithms implementation}

\subsection{The peak picking method (PPM)}

The PPM, sometimes referred as a peak-amplitude method or $3 \mathrm{~dB}$ method, is a single-input single output (SISO) and it is the simplest of the modal parameter estimation methods that works in the frequency domain [20, 21]. It consists of separating each single mode in order to determine their modal parameters. Thus, the natural frequency, damping factor and residues will be determined as follows, respectively:

$\omega_{r}=\max (F R F)$

$\zeta_{r} \cong \frac{\omega_{a}-\omega_{b}}{2 \omega_{r}}$

$A_{p q}^{r}=2|\tilde{\alpha}|_{p q} \omega_{r}^{2} \zeta$
Note that $\omega_{a}$ and $\omega_{b}$ are the frequencies on the halfpower points.

\subsection{Circle fit method (CFM)}

The CFM in the past was known as the Kennedy-Pancu method [4]; it is a single-input single output method and it works in the frequency domain. A detailed study has been established in Ref [22]. Similar to the PPM, it is designed to separate each single mode in the system. The concept of this method is to consider the FRF values in the vicinity of the resonance as a circle in the Nyquist plot. In fact, once the natural frequency and the damping factor were estimated, the diameter of circle is used to estimate the residues. According to the algorithm, the Circle-Fit method can be described by the following sequences:

- Selection of points to be used.

- Circle fitting based on these points and calculation of the fitting quality.

- Estimation of damping ratios and natural frequencies

- Calculation of multiple damping estimates and their mean and scatter.

- Determination of the modal constant.

$\omega_{r}=\max (\Delta \theta)$

$\zeta_{r}=\frac{\omega_{i+1}^{2}-\omega_{i}^{2}}{2 \omega_{r}\left(\omega_{i+1} \tan \left(\frac{\theta_{i+1}}{2}\right)+\omega_{i} \tan \left(\frac{\theta_{i}}{2}\right)\right)}$

$A_{p q}^{r}=2|\tilde{\alpha}|_{p q} \omega_{r}^{2} \zeta$

The robustness of this method has been demonstrated by several studies as given in Ref. [13-23].

\subsection{Least Square Complex Exponential Method (LSCEM)}

The LSCEM was introduced in 1979 [7], it is a single-input multi-outpout method (SIMO) and it works in the time domain. This method starts using the FRF receptance of a general MDOF system with a general viscous damping. Then, the impulse response function will be obtained by an inverse Fourier trasformation as follows:

$\alpha_{p q}(j \omega)=\sum_{r=1}^{2 N} \frac{A_{p q}^{r}}{j \omega-\lambda_{r}} \stackrel{\mathcal{F}^{-1}}{\longrightarrow} h_{i j}(t)=\sum_{r=1}^{2 N} A_{p q}^{r} e^{\lambda_{r} t}$ 
For the Ith sample.

$$
\begin{aligned}
h_{l}= & \sum_{r=1}^{2 N} C_{r} V_{r}^{\prime} I=0,1 \ldots, N-1 \\
& \text { with }\left\{\begin{array}{l}
C_{r}=A_{p q}^{r} \\
V_{r}=e^{\lambda_{r} t}
\end{array}\right.
\end{aligned}
$$

Equation (7) extended to the full data set of I samples, gives:

$\left[\begin{array}{ccccc}1 & 1 & 1 & \ldots & 1 \\ v_{1} & v_{2} & v_{3} & \ldots & v_{2 N} \\ \vdots & \vdots & \vdots & \vdots & \vdots \\ v_{1}^{\prime} & V_{2}^{\prime} & V_{3}^{\prime} & \ldots & v_{2 N}\end{array}\right]\left[\begin{array}{c}C_{1} \\ C_{2} \\ \vdots \\ C_{2 N}\end{array}\right]=\left[\begin{array}{c}h_{0} \\ h_{1} \\ \vdots \\ h_{2 N}\end{array}\right]$

Or, in compact form:

$[V][C]=[h]$

$[V]$ and $[C]$ are unknowns.

This equation can be solved by using Prony's Method [24], the roots $\lambda_{r}$ for an underdamped system always occur in complex conjugate pairs. It always exists a polynomial in $V_{r}$ of order / with real coefficients $\beta$, (called the Autoregressive coefficients) such as the following relation is verified:

$\beta_{0}+\beta_{1} V+\beta_{2} V^{2}+\cdots+\beta_{l} V^{\prime}=0$

After some steps explained in detail in the Ref [8], the following equation will be obtained:

$\left[\begin{array}{ccccc}h_{0} & h_{1} & h_{2} & \ldots & h_{2 N-1} \\ h_{0} & h_{1} & h_{3} & \ldots & h_{2 N} \\ \vdots & \vdots & \vdots & \vdots & \vdots \\ h_{2 N-1} & h_{2 N} & h_{2 N+1} & \ldots & h_{4 N-1}\end{array}\right]\left[\begin{array}{c}\beta_{1} \\ \beta_{2} \\ \vdots \\ \beta_{2 N-1}\end{array}\right]=-\left[\begin{array}{c}h_{2 N} \\ h_{2 N+1} \\ \vdots \\ h_{4 N-1}\end{array}\right]$

From Eq. (11), coefficients $\beta$ will be determined by using the single impulse response via a Least Square Method. Instead of using a single impulse response function (IRF), LSCEM estimates coefficients $\beta$ by using several IRF's, as follows.

$\left[\begin{array}{c}h_{11} \\ h_{12} \\ \vdots \\ h_{p}\end{array}\right][\beta]=-\left[\begin{array}{c}\widetilde{h_{11}} \\ \widetilde{h_{12}} \\ \vdots \\ \tilde{\widetilde{h}}\end{array}\right]$

In compact form,

$[H][\beta]=[\tilde{h}]$

Coefficient $\beta$ will be obtained by using pseudoinverse technique. Now coefficients $\beta_{0}, \beta_{1}, \ldots \beta_{2 N-1}$, are known and

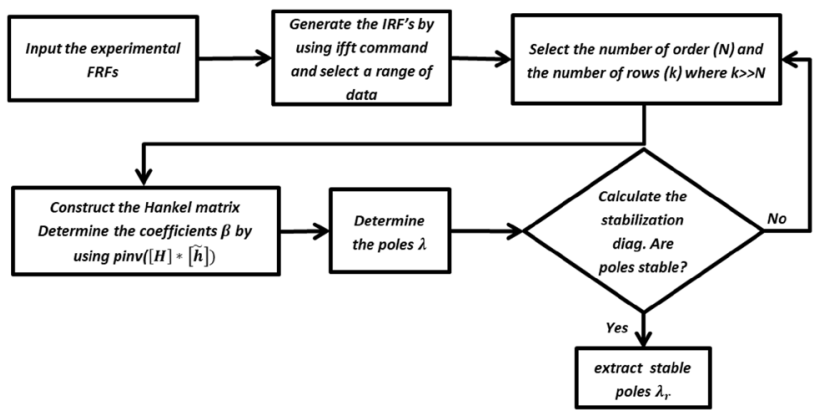

Fig. 2 Steps of the LSCEM implemented in Matlab ${ }^{\circledR}$

Eq. (10) can be solved to yield the $V_{r}$ roots. Then, poles $\lambda_{r}$ can be calculated as.

$\lambda_{r}=\frac{\ln \left(V_{r}\right)}{\Delta t}$

The mode shapes of the system can be calculated immediately by substituting $[V]$ in Eq. (9). The challenge of this method is to construct a good Hankel matrix $[H]$ where the number of columns represents the number of order and the number of rows are arbitrary. In order to minimize the Least Square Error, some studies supposed to consider a high number of rows $[21,25]$. In this study the number of rows will be determined by increasing the number of rows one at a time then the best number of rows will be found by observing the stabilization diagram [26]. If the stabilization diagram obtained was not clear enough, the range of time should be changed. A small diagram in Fig. 2 explains the procedure of the implementation in Matlab ${ }^{\circledR}$.

If it is not possible to obtain a clear stabilization diagram, the range of selected data must be changed and the same procedure mentioned above will be repeated. This method suffers when the damping is high as demonstrated in Ref [27].

\subsection{Eigensystem realization algorithm method (ERAM)}

The ERAM is a multi-input multi-output method (MIMO) and it works in the time domain [9]. It includes information not only from different output locations, but also from several input reference points on the structure. This method succeeded in dealing with the problem of missing one of the vibration modes from output responses, this occasionally happens following the application of a SIMO method. The Singular Values Decomposition (SVD) in this method can significantly reduce the effect of noise [7]. The ERAM is a very effective method for system identification by using Hankel matrix; further details about this method can be found in $\operatorname{Ref}[13,28]$. 
Let assume a state space dynamic system as follows:

$x(t+1)=\boldsymbol{A} x(t)+\boldsymbol{B} u(t)$

$y(t)=C x(t)+D u(t)$

Let consider an impulse force at $t=0$, and the initial condition equal to zero:

$\left\{\begin{array}{l}u(0)=1 \\ u(t)=0, \quad t>0 \\ D=0\end{array}\right.$

Hence, by iterating the system of Eq. (11) in time, the following parameters will be obtained.

$$
\begin{aligned}
x(0)=0 ; & \rightarrow y(0)=0 ; \\
x(1)=A x(0)+B u(0)=B & \rightarrow y(1)=C B ; \\
x(2)=A B ; \rightarrow y(2) & =C A B ;
\end{aligned}
$$

where $y(0), y(1), y(2) \ldots, y(t)$ are the so called Markov parameters.

By constructing the Hankel matrix $H_{0}$ of the Markov parameters as:

$\underbrace{H_{0}}_{(p x q)}=\left[\begin{array}{cccc}y(0) & y(1) & \ldots & y(p) \\ y(1) & y(2) & \ldots & y(p+1) \\ \vdots & \vdots & \ddots & \vdots \\ y(q) & y(q+1) & \ldots & y(p+q-1)\end{array}\right]$

And by apply the SVD to the matrix $H_{0}$

$$
[\underbrace{H(0)}_{p \times q}]=\underbrace{[\boldsymbol{U}]}_{p \times 2 N} \underbrace{[\Sigma]}_{2 N \times 2 N} \underbrace{[\boldsymbol{V}]^{T}}_{2 N \times q}
$$

And by shifting the Hankel matrix as follows:

$$
\underbrace{H_{1}}_{(p \times q)}=\left[\begin{array}{cccc}
y(1) & y(2) & \ldots & y(p+1) \\
y(2) & y(3) & \ldots & y(p+2) \\
\vdots & \vdots & \ddots & \vdots \\
y(q+1) & y(q+2) & \ldots & y(p+q+1)
\end{array}\right]
$$

After some manipulations [13], the identified discrete state-space $\hat{A}, \hat{B}$ and $\hat{C}$ can be written

$$
\begin{aligned}
& {[\hat{A}]=\left[\Sigma_{2 N}\right]^{-\frac{1}{2}}\left[\boldsymbol{U}_{2 N}\right]^{T}[H(1)]\left[\boldsymbol{V}_{2 N}\right]\left[\Sigma_{2 N}\right]^{-\frac{1}{2}}} \\
& {[\hat{B}]=\left[\Sigma_{2 N}\right]^{1 / 2}\left[\boldsymbol{V}_{2 N}\right]^{T}\left[E_{q}\right]} \\
& {[\hat{C}]=\left[E_{p}\right]^{T}\left[\boldsymbol{U}_{2 N}\right]\left[\Sigma_{2 N}\right]^{\frac{1}{2}}}
\end{aligned}
$$

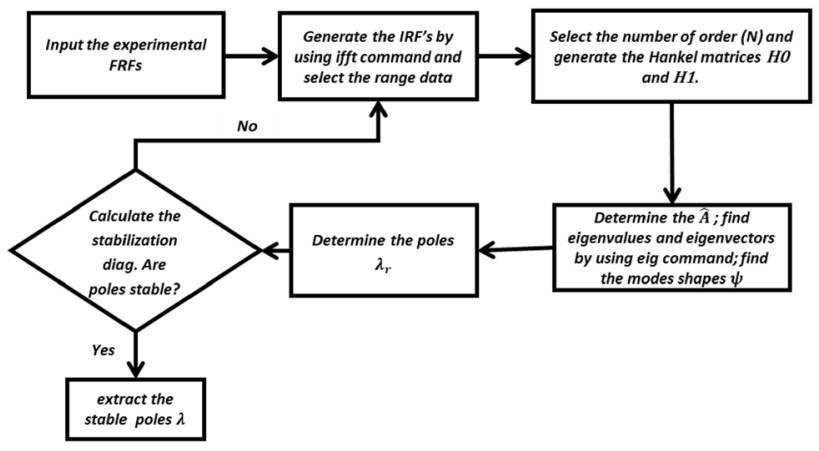

Fig. 3 Steps of the ERAM implemented in Matlab ${ }^{\circledR}$

In modal analysis, the goal is to determine matrices $\hat{A}$ and $\hat{C}$, where the eigenvalues of $\hat{A}$ consist of the complex conjugates poles of the system. From each pole the natural frequency and the damping ratio can be obtained. The mode shapes are related to matrix $\hat{C}$. By using matrix $\hat{A}$ and by solving the Eigen-problem, the mode shapes can be determined in terms of the physical coordinates of system:

$[\hat{A}]\left[\psi_{u}\right]=\Lambda\left[\psi_{u}\right]$

The transformation given by Eq. (11) must be used:

$\underbrace{\psi}_{p \times 1}=[\boldsymbol{C}]\left[\psi_{u}\right]$

$\Lambda=\left\{\Lambda_{1}, \Lambda_{2}, \ldots, \Lambda_{2 N}\right\}$ is the eigenvalues and $\left[\psi_{u}\right]$ is the eigenvector. The poles will be determined by the following formulae:

$\lambda_{r}=\frac{\ln \left(\Lambda_{r}\right)}{\Delta t}$

The ERAM was implemented in Matlab ${ }^{\circledR}$ by fixing a number of order $\mathrm{N}$ and generating the matrix $[\hat{A}]$ by iteration. For each iteration, matrix $[\hat{A}]$ was generated as a square matrix $(1 \times 1,2 \times 2, \ldots, N \times N)$. Then it was possible to extract the eigenvalues and the eigenvector. Furthermore, the poles were calculated by applying Eq. (24) and the physical mode shapes by using Eq. (20). Thus, the poles and the mode shapes were determined. The final step consists of plotting the stabilization diagram from which the stable poles are extracted. Figure 3 represents the main steps used to implement this method in Matlab ${ }^{\circledR}$.

\subsection{Rational fraction polynomial method (RFPM)}

This method works in the Frequency Domain [11], and it is a single-input single-output method (SISO), based on a multi degrees of freedom approach. The Rational Polynomial 


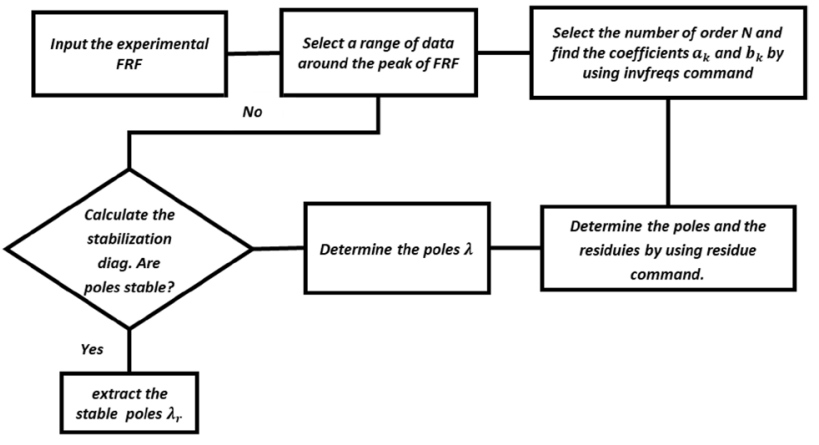

Fig. 4 Steps of the RFPM implemented in Matlab ${ }^{\circledR}$

Method (RFPM) is a special version of the general Curve-Fitting method, but it is based on the FRF which is expressed in the Rational fraction form [13]. Thus, the FRF requires an expression of the frequency response function as the ratio of two polynomials, with the roots of numerator permitting to determine the modal constant while the roots of the denominator yielding the poles (frequency, damping). Generally, the numerator and the denominator orders are independent one to each other. The denominator is considered as the characteristic polynomial of the system. By Curve-Fitting the FRF against the analytical form in Eq. (25), and then solving the roots of the numerator and the characteristic polynomials, the zeros and poles of the FRF can be determined.

$\alpha(j \omega)=\frac{\sum_{k=0}^{2 N-1} a_{k}(j \omega)^{k}}{\sum_{k=0}^{2 N} b_{k}(j \omega)^{k}}$

The curve fitting in the RFPM consists of determining the coefficients $a_{k}$ (with $k=0, \ldots 2 N-1$ ) and $b_{k}$ (with $k=0, \ldots 2 N$ ), in such a way the error between the analytical formulae (25) and the FRF is minimized over a chosen range; more information is given in Ref [29].

The RFPM was implemented in Matlab ${ }^{\circledR}$ by firstly selecting a small range of frequency and by "invfreqs" command the coefficients $a_{k}$ and $b_{k}$ were determined. Then, the poles and the residues were determined by using "residue" command. More information about "invfreqs" and "residue" command are included in Ref [30]. If it is not possible to obtain a clear stabilization diagram, the range of the frequency will be changed. Figure 4 describes the important steps that were used to implement the RFPM.

This method requires more time with respect to other methods to achieve a good estimation for the frequency and damping ratio.

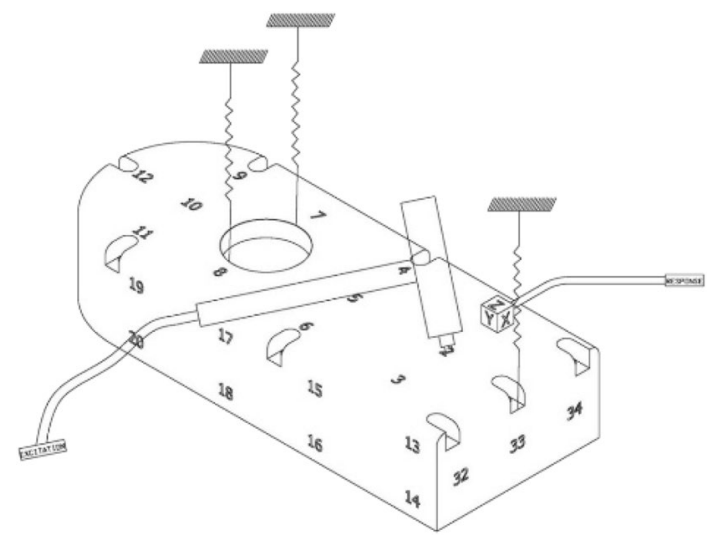

Fig. 5 Measurement set-up

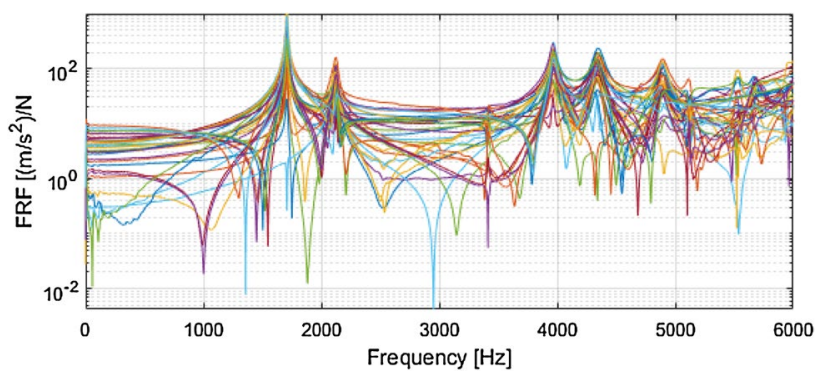

Fig. 6 Amplitude of all the measured frequency response functions

\section{Measurement and analysis}

Measurements were carried out on an aluminum carcass of gearbox with dimension of about $150 \mathrm{~mm}$ by $100 \mathrm{~mm}$ by $40 \mathrm{~mm}$ with carcass's thickness of $5 \mathrm{~mm}$. The carcass was supported by soft bungee cords fixed on a frame, in order to consider its dynamic behavior as a free-free condition. Figure 5 shows the set-up of the test measurement used in this study. As it can be seen, the periodic impulsive excitation is generated by using a hammer (PCB 068C04), while the response is measured by using PCB piezoelectric accelerometer (freq. range 1-10000 Hz). One accelerometer was positioned, and the system was excited by a hammer in 34 points. In particular, the response point was remained fixed during the tests, while the excitation changed from one measurement point to another in order to get the FRFs for all the considered points [31, 32]. The measured data were recorded using software LMS Test-Lab ${ }^{\circledR}[33]$ and the post processing was performed in Matlab ${ }^{\circledR}$ environment. The signals were acquired by using Nyquist frequency of $12,800 \mathrm{~Hz}$ and frequency resolution $\Delta f=1.5625 \mathrm{~Hz}$ compromising with the type of carcass gear box and the kind 
Table 1 Estimated natural frequencies, and corresponding normalized random errors, er, for the 5 methods

\begin{tabular}{|c|c|c|c|c|c|c|c|c|}
\hline & \multirow{2}{*}{$\begin{array}{l}\text { PPM } \\
f_{r}[H z]\end{array}$} & \multirow{2}{*}{$\begin{array}{l}\text { CFM } \\
f_{r}[\mathrm{~Hz}]\end{array}$} & \multicolumn{2}{|c|}{ LSCEM } & \multicolumn{2}{|l|}{ ERAM } & \multicolumn{2}{|l|}{ RFPM } \\
\hline & & & $f_{r}[H z]$ & $\operatorname{er}\left(f_{r}\right) \%$ & $f_{r}[\mathrm{~Hz}]$ & $\operatorname{er}\left(f_{r}\right) \%$ & $f_{r}[\mathrm{~Hz}]$ & $e r\left(f_{r}\right) \%$ \\
\hline Mode 1 & 1709 & 1707 & 1706 & 0.005 & 1706 & 0.002 & 1705 & 0.001 \\
\hline Mode 2 & 2125 & 2124 & 2125 & 0.067 & 2118 & 0.031 & 2123 & 0.021 \\
\hline Mode 3 & 3415 & 3414 & 3340 & 0.089 & 3413 & 0.013 & 3413 & 0.034 \\
\hline Mode 4 & 3964 & 3963 & 3962 & 0.076 & 3964 & 0.008 & 3967 & 0.001 \\
\hline Mode 5 & 4343 & 4343 & 4340 & 0.003 & 4341 & 0.023 & 4340 & 0.004 \\
\hline
\end{tabular}

Table 2 Estimated damping ratios, and corresponding normalized random errors, er, for the 5 methods

\begin{tabular}{|c|c|c|c|c|c|c|c|c|}
\hline & \multirow{2}{*}{$\begin{array}{l}\text { PPM } \\
\zeta_{r} \%\end{array}$} & \multirow{2}{*}{$\begin{array}{l}\text { CFM } \\
\zeta_{r} \%\end{array}$} & \multicolumn{2}{|c|}{ LSCEM } & \multicolumn{2}{|c|}{ ERAM } & \multicolumn{2}{|c|}{ RFPM } \\
\hline & & & $\zeta_{r} \%$ & $\operatorname{er}\left(\zeta_{r}\right) \%$ & $\zeta_{r} \%$ & $\operatorname{er}\left(\zeta_{r}\right) \%$ & $\zeta_{r} \%$ & $\operatorname{er}\left(\zeta_{r}\right) \%$ \\
\hline Mode 1 & 0.27 & 0.23 & 0.29 & 0.08 & 0.28 & 0.616 & 0.29 & 0.042 \\
\hline Mode 2 & 0.55 & 0.51 & 0.53 & 0.069 & 0.51 & 0.125 & 0.65 & 0.029 \\
\hline Mode 3 & 0.44 & 0.53 & 0.45 & 0.073 & 0.3 & 0.190 & 0.25 & 0.131 \\
\hline Mode 4 & 0.45 & 0.47 & 0.53 & 0.04 & 0.54 & 0.132 & 0.47 & 0.010 \\
\hline Mode 5 & 0.74 & 0.74 & 0.72 & 0.029 & 0.76 & 0.012 & 0.84 & 0.008 \\
\hline
\end{tabular}

of constraint condition. An exponential window for the force signals is used in order to decrease the leakage. The input autopower-spectra, output power-spectra and crosspower-spectra are estimated and reserved for each measurement location. In addition, the FRFs are determined by using $H_{v}$ estimator [33]. The coherence function is controlled as an on-line check of data quality. The periodic response was calculated by using the synchronous averaging method as reported in [19] with the aim of obtaining a good estimation of the frequency response function.

In detail, 34 periodic responses were selected to have a better estimation of the modal parameters, i.e. natural frequencies and damping ratios. The method used to make the EMA is the traditional procedure in which both excitation and response are measured together to obtain the accelerance. Figure 6 shows all the FRFs measured, in total 34 functions are plotted.

Five methods used for the estimation of modal parameters are shown in this work. At the beginning, the Peak Amplitude and Circle Fit were the simplest methods to be applied to calculate the first estimation as their implementation is easier with respect to the other methods. Once the results were obtained by these two methods, they were compared with the results of several methods that, according to literature, are considered more accurate; these methods are the LSCEM, ERAM, and RFPM.

A stabilization diagram was constructed for the last three methods to identify the physical modes from the computational modes. Normally, a tolerance, in percentage is given for the stability of each modal parameters.

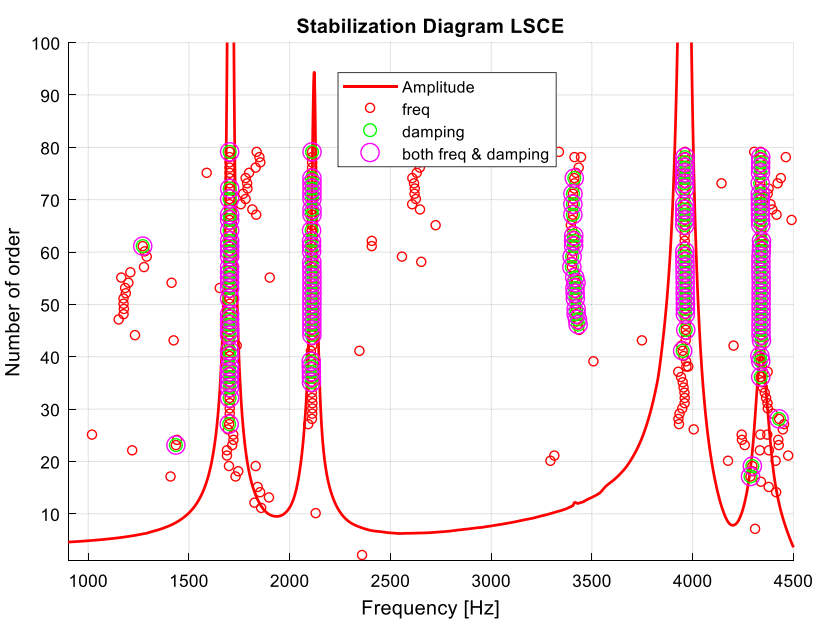

Fig. 7 Stabilization diagram extracted by LSCE

\section{Results and discussion}

The natural frequencies and damping ratios were extracted for the first 5 modes by the methods described in Sect. 2, in the following manner. In the LSCEM, ERAM and RFPM, all modes fulfilling the stabilization criteria with tolerance $1 \%$ for the natural frequencies, $5 \%$ for the damping ratios and the MAC [34] values greater than $90 \%$.

In the LSCEM the stable poles that have stability in frequency and damping in the stabilization diagram were plotted. The LSCEM stability diagram shows that the vectors are not engaged, because the non- physical 


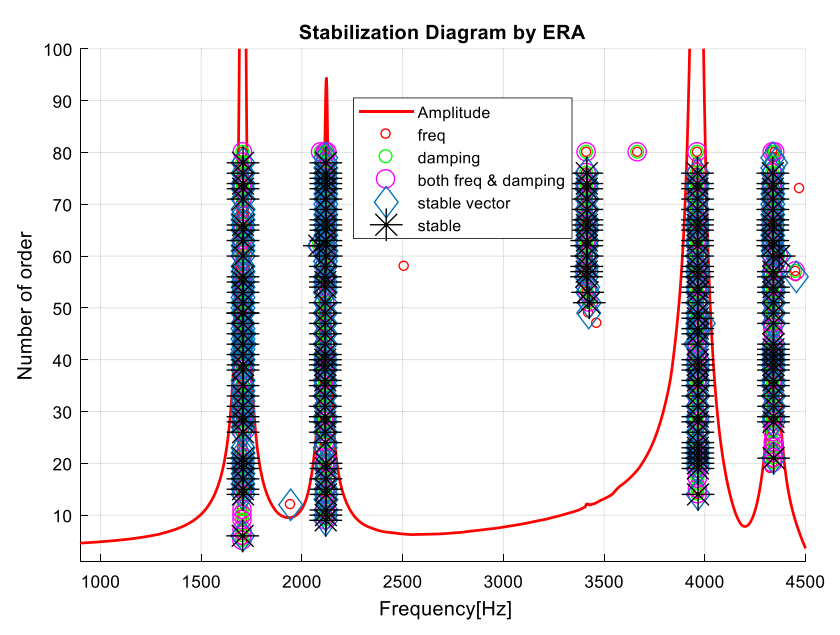

Fig. 8 Stabilization diagram extracted by ERAM

poles appear with the increase of the number of orders. Consequently, it will be hard to reach a good estimation of the mode shapes. In the ERAM the stability of vector and the stable poles were schemed. In order to compare the mode shapes between the iteration, the Modal Assurance Criterion (MAC) was used to detect the stability of vectors. When the RFPM method is applied, the stability of the vector can be rarely found because as the number of orders increases the ill conditioning problem starts to appear. Therefore, its plot was eliminated in the stabilization diagram of the RFPM.

The results are summarized in Tables 1 and 2, where the average value of poles and the corresponding scatter are listed and schemed in the Figs. 10 and 11. The scatter was calculated as the normalized random error er (standard deviation divided by the average value) for LSCEM, ERAM and RFPM. It is important to mention here that the minimum number of pole estimates of each mode was 12 .

The estimation of the stable poles can be seen in Figs. 7, 8 and 9. Figures 7 and 8 show the stabilization diagrams extracted by LSCEM and ERAM, respectively, with a number of order 80 . In both cases, the presence of 5 physical modes can be detected in the frequency range [0 4500] Hz. It can be noticed that the stable poles start to appear when the number of order surpasses 26 for the LSCEM (Fig. 7). In Fig. 9 all modes were isolated, and for each mode a stabilization diagram was established with a number of order 20 . When the frequency bandwidth is narrowed around the Peaks, these modes immediately appear when the number of order exceed one, and their estimation becomes more accurate.
It is evident from Table 1 that the natural frequencies of all modes determined by these methods were very similar and their errors er were very low; thus, the difference between the method results is insignificant. Regarding the damping factor, it can be noted in Table 2 that the results were very distant, and it is difficult to detect a good damping estimation. The comparison between the PPM and CFM and the other methods in Table 2 shows that their results were slightly different, while some similarity can be noticed on several modes. It can be deduced that although the PPM and CFM are generally considered simple and very basic as described in Sect. 2, they managed to give good results in comparison to the other methods. Therefore, these two methods can be very useful as a first estimation because they provide good results for the major part of the modes.

In this study, the error values er is an indicator of how good a method works. When the error is low, the stability of the poles is high in the stabilization diagram, hence the method can be considered reliable. In this context, a comparison was made between RFPM, ERAM and LSCEM in Figs. 10 and 11; it was clear that the RFP was the method that presented the lowest values of error which make it the best method among the three studied. The only issue here is that the error obtained by RFPM was the lowest on all the modes except the third mode, where it has a value $(0.15 \%)$ greater than the error given by the LSCEM and almost equal to the one given by the ERAM. This error can be due to the noise present in the experimental FRF.

\section{Conclusions}

This paper presents a comparison between several methods of experimental modal analysis for the detection of the frequency and damping ratio values. The results achieved during the study serve to understand the factors affecting the accuracy of the frequency and the damping in respect to the method in use. It can be observed that the natural frequency values obtained by all methods were very similar and the differences between the results were insignificant.

It was revealed that it is convenient to use the Peak Picking Method and Circle Fit Method as a first estimation; these methods succeeded in giving good estimation of the natural frequency and damping. They are also simple to use and fast to implement. 
(a)

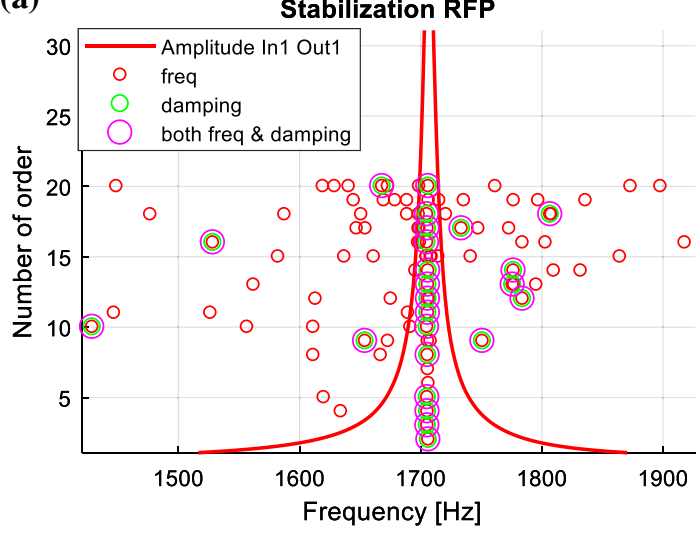

(c)

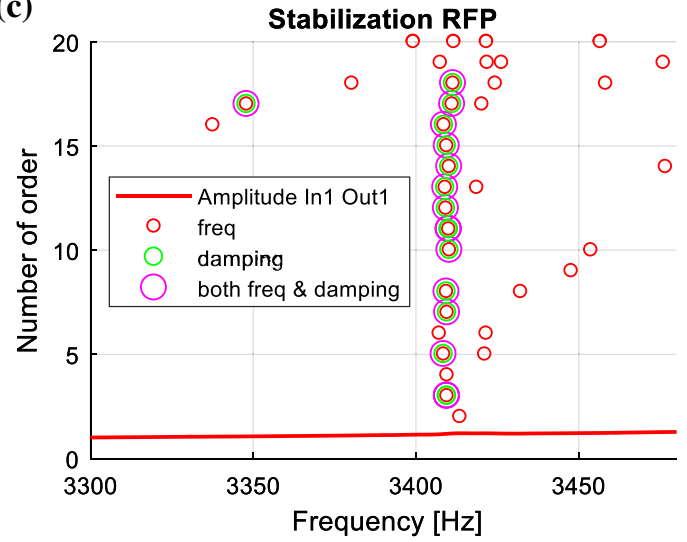

(b)

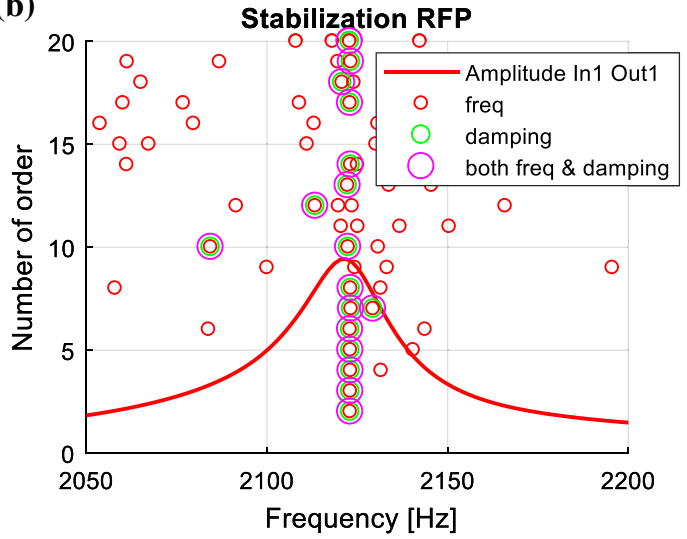

(d)

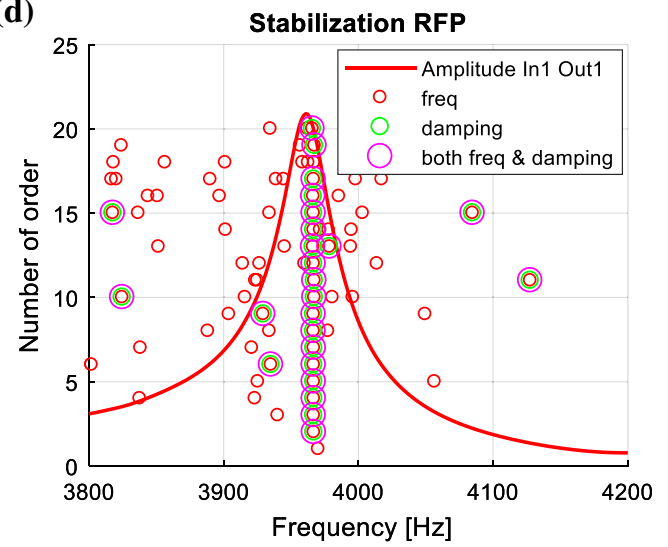

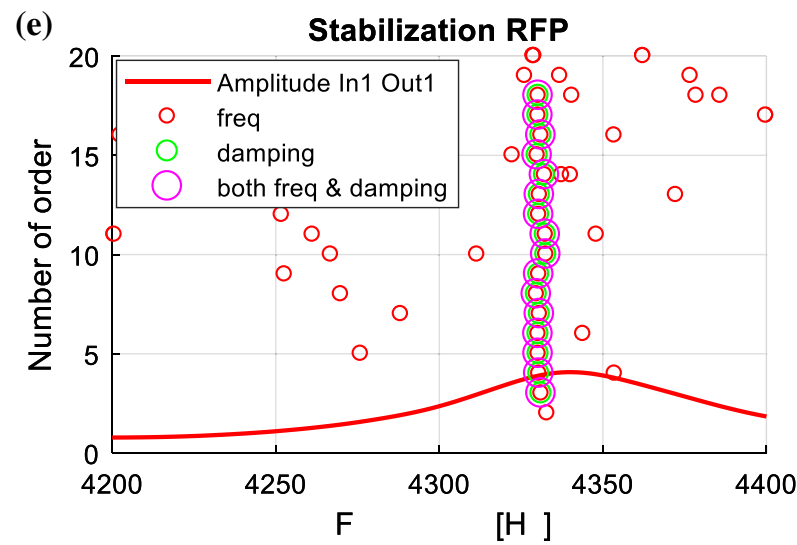

Fig. 9 a Stabilization diagram close to the first mode; $\mathbf{b}$ second modes; $\mathbf{c}$ third mode; $\mathbf{d}$ fourth mode; e fifth mode

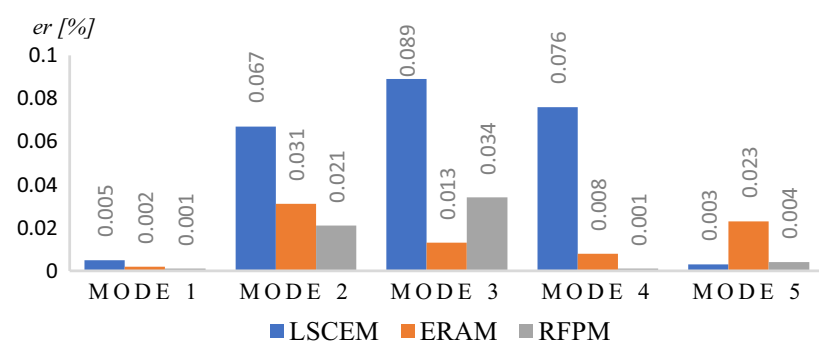

Fig. 10 Scatter of frequency for each mode, determined by LSCEM, ERAM and RFPM
Regarding the other methods, if the stability of poles dispersed in the stabilization diagram was taken into consideration, the Rational Fraction Polynomial method did the best job in detecting the damping and frequency values. This method presented the least scatter on all modes except on the third, probably due to the noise present in the measured frequency response function. The results obtained by the LSCEM and the ERAM were reasonable for both frequency and damping. The 


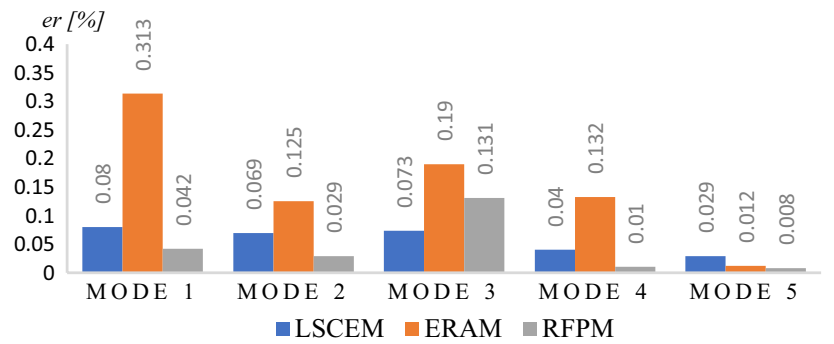

Fig. 11 Scatter of damping for each mode, determined by LSCEM, ERAM and RFPM

scatter was found slightly greater in the ERAM than in the LSCEM.

In addition, it has been noted by following this procedure of implementation. The fast method to extract the natural frequencies and the damping ratios was the ERAM next come the LSCEM then the RFPM.

\section{Compliance with ethical standards}

Conflict of interest The authors declare that they have no conflict of interest regarding the publication of this paper.

\section{References}

1. Rieger NF (2003) The relationship between finite element analysis and modal analysis, stress technology incorporated. Rochester, New York

2. Zhou W, Chelidze D (2008) Generalized eigenvalue decomposition in time domain modal parameter identification. J Vib Acoust ASME 130(1):011001. https://doi.org/10.1115/1.2775509

3. Cakir F, Habib U (2015) Experimental modal analysis of brick masonry arches strengthened prepreg composites. J Cult Herit $16: 284-292$

4. James MWB, Alison R, James B, Alessandro A, Emma H, Peter D (2018) Experimental modal analysis of British rock lighthouse. Mar Struct 62:1-22

5. Diogo M, Julio MMS (2014) A contribution to the modal identification of the damping factor based on the dissipated energy .In: Proceedings of the 9th international conference on structural dynamics Portugal 30 June-2 July

6. LMS International (2005) The LMS theory and background book-analysis and design. Manual of test. Lab revision 5. LMS International, Leuven

7. Brown DL, Allemang RJ, Zimmerman RD, Mergeay M (1979) Parameter estimation modal techniques for modal analysis. SAE technical no. 790221, 15-24

8. Juang JJ (1994) Applied system identification. Prentice-Hall, Englewood Cliffs
9. Juang JN, Pappa RS (1985) An eigensystem realization algorithm for modal parameter identification and model reduction. J Guid Control Dyn 8:620-627

10. El-Kafafy M, Guillaume P, Peeters B, Marra F, Coppotelli G (2012) Advanced frequency- domain modal analysis for dealing with measurement noise and parameter uncertainty. In: Conference proceeding of the 30 th IMAC, a conference on structural dynamics, Bethel, CT, USA, 5. Springer, pp 179-198

11. Richardson $\mathrm{MH}$, Formenti DL (1982) Parameter estimation from frequency response measurements using rational fraction polynomials. In: Proceedings of the 1st IMAC conference, Orlando, Florida, November 1982, pp 167-181

12. Brincker R, Ventura CE (2015) Introduction to operational modal analysis. Wiley, London

13. Silva JMME (1978) Measurements and application of structural mobility data for the vibration analysis of complex structures. Ph.D. thesis, Imperial College of Science and Technology, London, UK

14. Davis D, Abrams M, Brault J (2001) Fourier transform spectrometry, 1st edn. Academic Press, ebook 9780080506913

15. Mallat S (2008) A wavelet tour of signal processing, 3rd edn. Academic Press, ebook 9780080922027

16. Douglas $L(2009)$ The discrete Fourier transform, part 4: spectral leakage. J Object Technol 8:7

17. Fahey SO'F, Pratt J (1998) Frequency domain modal estimation techniques. Exp Tech 22:33-37

18. Fahey SO'F, Pratt J (1998) Time domain modal estimation techniques. Exp Tech 22:45-49

19. Ford R, Randall RB, Wardrop T (2002) Updating modal properties from response-only measurements on a rail vehicle. In: International conference on noise and vibration engineering, Leuven, September 16-18

20. Ewins DJ (2000) Modal testing: theory, practice and application, 2nd edn. Wiley, New York

21. Fasana A, Marchesiello F (2006) Meccanica Delle Vibrazioni. C. L. U. T, Torino

22. Maia NMM (1985) Interference criteria in modal analysis identification. M.Sc. Thesis, Technical University of Lisbon, Portuguese

23. Robb DA, Ewins DJ, Maia NMM (1985) Modal tests on the end windings of an electric motor. Imperial College of Science and Technology, London

24. Prony R (1795) Essai expérimental et analytique sur les lois de la dilatabilité des fluides de la vapeur de l'alkool, à différentes températures. Journal de l'École Polytechnique Floréal et Plairial, 1, 22:24-76

25. Helyen W, Lammens S, Sas P (1998) Modal analysis theory and testing. Katholieke University, Leuven

26. Allemang RJ, Philips AW (2004) The unified matrix polynomial approach to understanding modal parameter estimation an update structure dynamic. Research laboratory, University of Cincinnati, Cincinnati

27. Brillhart SR, Mikulcik EC (1988) Comparison of modal parameter estimation methods for highly damped structures. In: Proceedings of IMAC VI, Kissimmee, Florida, pp 705-711

28. Juang JN, Pappa RS (1986) Effect of noise on modal parameters identified by eigensystem realization algorithm. J Guid Control Dyn 9(3):294-303

29. Francisco $M$, Marriaga ME, Pérez TE, Piñar MA (2019) Coherent pairs of bivariate orthogonal polynomials. J Approx Theory 245:40-63

30. Code of Matlab Software's "invfreqs" https://it.mathworks.com/ help/signal/ref/invfreqs.html and "residue" https://it.mathworks. com/help/matlab/ref/residue.html function, Mathworks 
31. Mucchi E (2013) On the sweet spot estimation in beach tennis rackets. Measurement 46(4):1399-1410. https://doi. org/10.1016/j.measurement.2012.12.014

32. Mucchi E (2012) Experimental evaluation of modal damping in automotive components with different constraint conditions. Mecc Int J Theor Appl Mech AIMETA 47(4):1035-1041

33. LMS Test.Lab (2004) Theory and background book. LMS International, Dubai
34. Allemang RJ (2003) The modal assurance criterion: twenty years of use and abuse. Sound Vib 37:14-23

Publisher's Note Springer Nature remains neutral with regard to jurisdictional claims in published maps and institutional affiliations. 